\title{
The implications of calorie restriction on longevity and health span
}

\begin{abstract}
Calorie Restriction (CR) is a defined intervention tool currently being studied as an effort to achieve numerous health benefits and increase longevity. Currently, there is a large body of research that indicates a multitude of health benefits and positive associations; however, there is also a plethora of opposing research. Through investigation of epigenetics, the efficacy of calorie restriction is being examined to provide insight into its potential benefits. The alterations to metabolic pathways may also reveal a reduction in inflammation and oxidative stress, markers of agingrelated disease. In addition, both animal and human studies take a closer look at the effects of calorie restriction on preservation of mitochondria and reduction of chronic disease. The idea of amelioration of these symptoms is extremely attractive; a body of literature suggests the possibility of mimicking the potential effects without calorie restriction at all. Regardless, as this new topic emerges, researchers' conclusions come closer and closer to making clinical recommendations.
\end{abstract}

Volume 2 Issue 5 - 2015

\author{
Zachari Breeding \\ Drexel University College of Medicine, USA
}

Correspondence: Zachari Breeding, Drexel University College of Medicine, USA, Email zacharibreeding@gmail.com

Received: December 23, 2014 | Published: June 16, 2015

Keywords: calorie restriction, aging, nutrition, obesity, chronic disease, longevity, health, inflammation

Abbreviations: CR, calorie restriction; SIRT, sirtuin; NAD, nicotinamide adenine dinucleotide; NADH, nicotinamide adenine dinucleotide hydrogenase; DNA, deoxyribonucleic acid; NF, necrosis factor; IL, interleukin; PPAR, peroxisome proliferatoractivated receptor; NIA, national institute of aging; ROS, reactive oxygen species; IKK, i kappa b kinase; AP, activator protein; PGC, peroxisome proliferator-activated receptor gamma cofactor; WNPRC, wisconsin national primate research center; IF, intermittent feeding; NRIP, nuclear receptor-interacting protein; AMPK, adenosine monophosphate-activated protein kinase

\section{Introduction and overview of calorie restriction}

As early as the $16^{\text {th }}$ century, humans have been fascinated with the idea of "the fountain of youth." Since then, people have made great attempts at lengthening their life span, all the while hoping to maintain optimal health until expiration. Recent studies have suggested calorie restriction $(\mathrm{CR})$ as a possible modality to delay or prevent the onset of many aging-related conditions with increased longevity and health span. CR is characterized by a $10-40 \%$ reduction in intake of a nutrient-rich diet. This restricted diet has been used extensively to research the effects intake has on longevity of life as well as health span. For the purposes of this paper, the term "health span" is used to characterize the duration of time in which a participant undergoing $\mathrm{CR}$ is of adequate or optimal health.

To date, there is a gamut of literature available investigating the effect CR has on overall health. As most of these are animal studies, it can be understood that the anatomical and physiological similarities between humans and non-human primates make the latter suited to provide insight into human aging; however, significant outcomes have been discovered from rat studies. The mechanisms behind the action of $\mathrm{CR}$ are numerous, including nutrient-responsive signaling molecules; effects on genes, hormones and neurons; and the reduction of reactive oxidative species, therefore decreasing inflammation and the onset or progression of chronic diseases associated with aging. In this review of current research, we will examine the significant effects of $\mathrm{CR}$ on longevity of life and health span in human as well as animal studies.

\section{The relationship with SIRT- I}

SIRT-1, known as sir2 in animal studies, is a protein that in humans is encoded by the SIRT-1 gene and is also a NAD-dependent deacetylase. ${ }^{1}$ Sir 2 has been shown to have NAD-dependent histone deacetylase activity, which contributes to cellular regulation and reaction to stressors; and therefore, longevity., ${ }^{2,3}$ Therefore, sir2 has been suggested to connect cellular energetics to lifespan via NAD which is used in metabolic reactions. In CR SIRT-1, increases which is related to a cascade of antithrombotic effects, DNA repair, genomic stability, apoptosis, growth and differentiation, inflammatory inhibition and glucose/insulin homeostasis. By seemingly attenuating the effects of metabolic syndrome, this increase in SIRT-1 can promote the delay in onset and progression of chronic diseases associated with this condition such as diabetes, cardiovascular disease, myocardial infarction, stroke and renal failure. ${ }^{3,4}$

Additionally, CR was associated with Sir2 via an alteration in the NAD/NADH ratio and changes in respiration activity. In Lin SJ et al., ${ }^{5}$ Sir2 was required to increase respiration, which led to an increased need of Sir2; this activity was concluded to have a connection to longevity by amplifying overall Sir2 activity. ${ }^{5}$

Furthermore, CR was shown to attenuate the up regulation of nuclear factor (NF)-kB $\mathrm{kB}^{57}$, a transcription factor that induces expression of tumor necrosis factor in white adipose tissue (used for fat storage in humans) and the production of pro-inflammatory cytokines such as interleukins IL-1, IL-2 and IL-6 in immune cells. Regarding this, SIRT1 was shown to down regulate (NF)-kB; ${ }^{59}$ creating the speculation that increased levels of SIRT-1 from CR may result in insulin sensitivity and reduction in inflammation. White adipose tissue is regulated by peroxisome proliferator-activated receptor ((PPAR)y); specifically, 
the a P2 gene encodes a protein to assist with fat storage. In Picard F et al, SIRT-1 downregulated this activation in mice during a period of slight starvation (similar to the effects of CR); this promoted fat mobilization into the blood. Thus, this suppression of adipogenesis may alter the hormonal levels linked to aging. ${ }^{6,7}$

SIRT-1 may play a role in preservation of neuronal integrity. In animal studies, CR was found to have an effect against degeneration of neurons associated with Huntington's disease, Alzheimer's disease, Parkinson's disease and stroke. It was also reported that CR stimulated neurogenesis and synaptic plasticity, which might increase the ability of the brain to resist cognitive decline. ${ }^{8}$ A recent report from Bordone et al., ${ }^{3}$ discussed the apparent need of SIRT- 1 to achieve a neuroprotective effect. The mechanism of this is still unknown, but it is suggested that the subsequent activation of genes encode proteins, which move down axons, protecting them. ${ }^{3}$

\section{Calorie restriction and oxidative stress}

Aging is accompanied by changes in gene expression involved with oxidative stress, which may be mitigated by CR. Free radicals, known as reactive oxygen species (ROS), are a highly reactive species that contain an unpaired electron on the cellular level causing oxidative stress. There are several causes of development of free radicals including lack of antioxidant intake (vitamins A, C, E) and environmental factors, among others; there is also association with the development of advanced glycation end products, which lead to a cascade of age related conditions including diabetes, cardiovascular disease, cataracts, stroke, kidney disease and liver failure. ${ }^{9,10}$ Additionally, unpaired electrons that leak out of the electron transport chain can cause ROS development. This can lead to damage in lipids, protein and DNA; generation of ROS is associated with increased incidence of atherosclerosis, rheumatoid arthritis, neurodegenerative disorders and cancer. ${ }^{11,12}$

As discussed earlier, CR has been studied to suppress proinflammatory cytokine production. In maintaining this well-balanced redox state, $\mathrm{CR}$ has been found to suppress oxidative stress through two important mechanisms. It has been established that inhibition of NF$\mathrm{kB}$ reduces inflammation, but also has an association with mitigation of oxidative stress. The phosphorylation of IKK during aging degrades proteins (including IkB) that enable NF-kB's binding activity to also increase. CR has been shown to suppress this key enzyme [IKK], which in turn upregulates $\mathrm{IkB}$ and thus mitigates activation of NF$\mathrm{kB}$. In this, IKK was associated with an increase in ROS, though the underlying mechanism is still unknown. Furthermore, upregulation of transcription factor AP-1 has mechanisms related to inflammation similar to NF-kB; its regulation derives from gene transcription levels. ${ }^{13} \mathrm{CR}$ was found to prevent activation of this protein in rats through inhibition of phosphorylation of regulatory factors. ${ }^{13}$ Because of the association between the inappropriate activation of prooxidative NF-kB and AP-1 with age-related diseases, it is integral to inhibit these processes in order to increase likelihood of longevity.

Since the mitochondria are the site where most oxidative stress in the form of ROS exists, it is important to take a closer look at how CR affects this key region in the cell. ${ }^{14}$ Mitochondrial dysfunction increases with age; it can implicate the process in which a plethora of diseases develop: cataracts, diabetes, obesity, inflammation, hypertension, atherosclerosis, Parkinson's disease, Alzheimer's disease, nervous system ischemia, sarcopenia, chronic fatigue syndrome and Fanconi's syndrome.$^{15}$ Because respiratory activity (mitochondrial biosynthesis) enhances cell longevity, this process plays a major role in the lifespan of an organism. Mitochondrial dysfunction can develop from nonfunctional complexes from within the electron transport chain (namely age-related downfall of complex IV), DNA damage and/or the accumulation of nonfunctional proteins resulting from ROS. ${ }^{15,16} \mathrm{In}$ $\mathrm{CR}$ animals, it has been demonstrated that even a short duration of two weeks had a significant effect in improved mitochondrial function. ${ }^{17} \mathrm{~A}$ suggested explanation of this could be the correlation of CR alteration with mitochondrial membrane fatty acid composition, which increases functionality. In expression and activation of transcription factor PGC-1 $\alpha$ leads to increased mitochondrial mass and biogenesis through a variety of mechanisms; in addition, this expression is shown to be maintained with aging (Figure 1)..$^{18,19}$

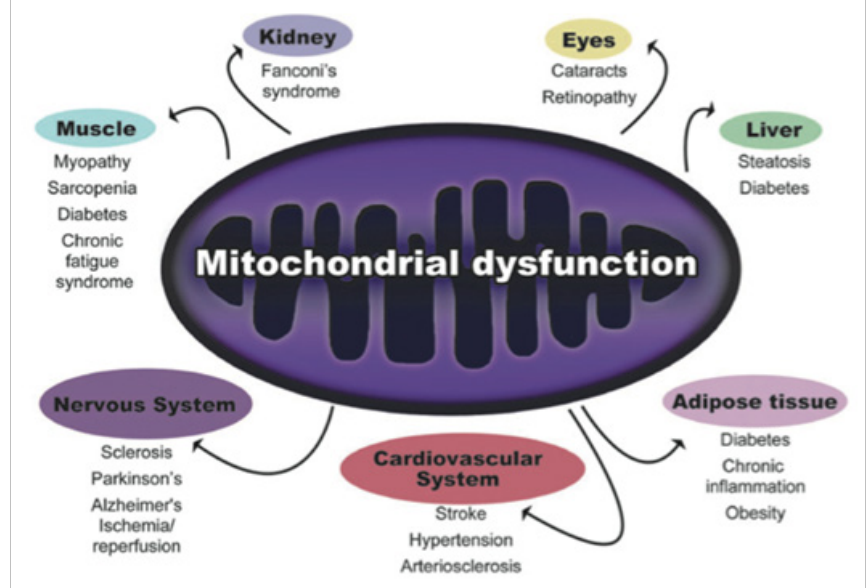

Figure I portrays the many roles of CR on improved mitochondrial function.

Because of the innate complications associated with long term randomized human trials and CR including lack of dietary compliance and required length of study duration for participants, there are few well designed human trials that exist to date associating CR and longevity. However, in one randomized controlled human trial, known as the CALERIE study, the duration was six months. This study selected and randomized 48 overweight (BMI 25-30) human participants aged over 50years (men) and over 45years (women) into four intervention groups for a six month period: 1) control, 2) $25 \%$ calorie restriction, 3) $12.5 \%$ calorie restriction plus exercise, 4 ) very-low calorie diet ( $890 \mathrm{kcal}$ per day) until 15\% weight reduction was reached followed by a weight maintenance diet. In this study, meals were provided, weekly group meetings were initiated and an exercise regimen was quantified. The study aimed to measure weight, body fat and total energy expenditure via doubly labeled water, indirect calorimetry and metabolic chamber. In addition, participants underwent numerous metabolic tests during a 5-day inpatient stay at the research center using fasting blood samples.

The study concluded that weight decreased in all groups: 1) -1.0 , 2) $-10.4 \%, 3)-10.0 \%, 4)-13.9 \%(p<.001)$. Fat mass was significantly reduced in all intervention groups compared to controls: (2) $-24 \%$, (3) $-25.0 \%$, (4) $-32.0 \%(\mathrm{p}<.001)$. Measured and adjusted 24 -hour energy expenditure significantly reduced in all intervention groups $(\mathrm{p}<.01)$. Fasting insulin levels were significantly reduced in all intervention groups by month $6(\mathrm{p}<.01)$. DNA damage was reduced in all intervention groups by month $6:$ (2) $-0.56 \mathrm{AU}$, (3) $-0.45 \mathrm{AU}$, (4) $-0.35 \mathrm{AU}(\mathrm{p}<.005)$. The CALERIE study is integral to begin the path to discovering the real association between effects of $\mathrm{CR}$ on health span and oxidative stress in humans. ${ }^{20} \mathrm{CR}$-induced hypoinsulinemia has been found to block phosphorylation of a major age-related 
transcription factor (FOXO1) that regulates NF-kB translocation through activation of age-associated kinases. ${ }^{21,22}$ The downregulation of this pathway may lead to profound increases in longevity alone. ${ }^{23}$ The attenuation of DNA fragmentation not only indicates reduced DNA damage, but also reduced oxidative stress during this short stint of CR in this group of participants. ${ }^{20}$ However, due to the short duration of this study, it may not be adequate to validate the hypothesis that $\mathrm{CR}$ extends life span. It can also be conjectured that CR may stimulate ghrelin levels, resulting in suppression of leptin, increased hunger drive and food consumption rebound. ${ }^{24}$

\section{Effect of calorie restriction on chronic disease risk}

Though the CALERIE study yielded observations that indicated a reduction in characteristics associated with chronic disease risk, the short duration of the study limits the definitiveness of this correlation. The attenuation of chronic disease risk is integral to increased longevity and health span; to date, the associations found between chronic disease indicators and CR have been promising yet controversial.

In a compelling study, Colman and associates performed a 20year longitudinal epidemiological study on rhesus monkeys and CR. This study assessed mortality, age-related survival and incidence of disease. In their analysis of 76 animals, it concluded that $37 \%$ of controls died of age-related causes compared to $13 \%$ of the $\mathrm{CR}$ group. The intervention group (CR) was significant for increasing survival with Cox regression $(p=.03)$ with HR 3.0; this indicated that the control group had three times the rate of death compared to the intervention group. The incidence of chronic diseases including cancer, cardiovascular disease and glucoregulatory impairment were decreased in the CR group compared to controls (Figure 2). Regarding these conditions, incidence of cancer was reduced by $50 \%$, incidence of cardiovascular disease was reduced by $50 \%$ and there was no incidence of diabetes in the CR group. Age-related disease reduction was statistically significant $(\mathrm{p}=.008)$ and more likely to occur in controls than the intervention group.

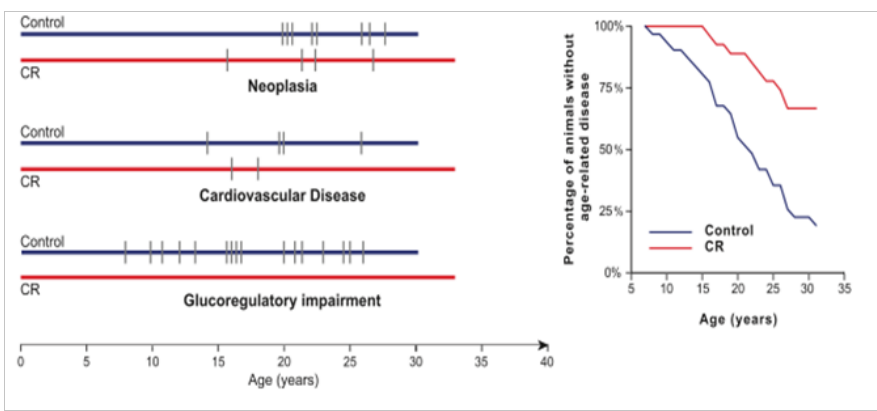

Figure 2 Percentage of animals without age related disease.

Furthermore, age-related sarcopenia was attenuated in CR animals and confirmed via XR absorptiometry. This intervention group also appeared to be younger (Figure 3). The study determined that CR significantly altered the aging effect in the intervention group and further promoted survival of this species by preservation of gray matter, cortex and lobe volume within the brain. Results of cardiovascular aging were supported by human studies on long-term CR who showed reduced risk. ${ }^{25}$

In a similar study from the National Institute of Aging (NIA) in coalition with the Wisconsin National Primate Research Center (WNPRC), the impact of CR on rhesus monkeys was examined for over 20years in order to document any potential health effects. The study included approximately 60 males and 60 females' ages 15 20 and 20-25years (grouped "young" and "old") with a maximum lifespan in captivity of approximately 40years. Study design was similar to the Colman and associates report, however this study continues past 2012. At the time of this article, four CR monkeys and one control have lived beyond 40years. However, old-onset CR monkeys did not live significantly longer than controls. CR has not increased mean or maximum lifespan compared to controls. Males did live significantly longer than females $(p=.0003)$, but neither benefited from CR. Furthermore, CR was found to be associated with reduction in triglycerides $(\mathrm{p}=.026)$, cholesterol $(\mathrm{p}=.02)$ and fasting glucose $(\mathrm{p}=.04)$ compared to controls. In addition to these findings, plasmafree isoprostane (an indicator of oxidative stress) was significantly higher in control than CR monkeys $(p=.009)$. However, decreased immune function was found in CR monkeys over controls. The study concludes that although $\mathrm{CR}$ is associated with several improved metabolic, cardiovascular and inflammatory markers, there was no link to longevity. ${ }^{26}$

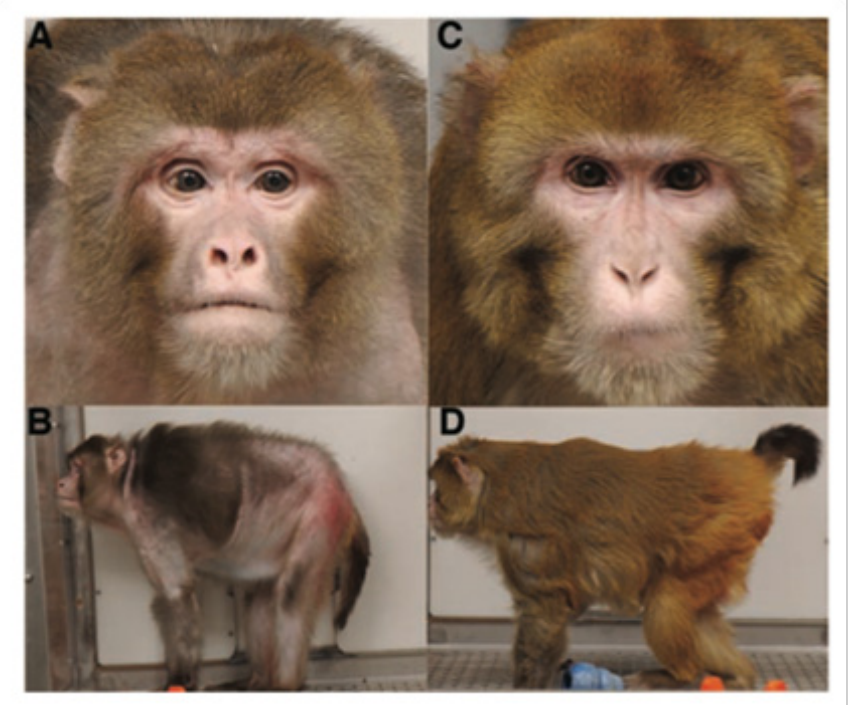

Figure 3 Animal appearance in old age (A and B) photographs of a typical control animal at $27.6 y$ ears of age (about the average life span). $C$ and $D$ photographs of an age-matched animal on CR.

\section{Calorie restriction mimetics}

Because CR in humans is a life-long commitment that may result in reduced compliance upon extended durations, several studies exist investigating the efficacy of singular strategies created naturally by the effects of $\mathrm{CR}$ in an effort to mimic the benefits without caloric restriction. ${ }^{27}$

Intermittent feeding (IF) is a pattern of eating easily described by providing food ad libitum but only every other day. In an animal study, this process of intermittent fasting resulted in more chow consumed per day with benefits related to longevity and health span similar to CR. However, this benefit was concluded to be associated with fewer calories provided overall and not necessarily the feeding pattern. ${ }^{28}$ Despite the fact that this process can result in weight loss, there is no realistic method of recommending this as an effective strategy in humans. Additionally, IF and CR have both been linked to abnormally high intake once food has been offered due to a state of acute and chronic hunger. ${ }^{29,30}$ This argument is one that stresses the importance of a nutrient-rich diet when undergoing a $\mathrm{CR}$ regimen. 
Another mimetic considered is liposuction. This technique does not deliver the same effect as CR in reducing white adipose tissue and may cause further detriment by allowing for enlargement of remaining fat cells $;{ }^{31}$ however, in rat studies, surgical removal of visceral fat resulted in significant health and longevity benefits independent of $\mathrm{CR}$ due to reduction of fat depots associated with increased morbidity and mortality. ${ }^{32}$

Resveratrol is a popular polyphenol found in red grapes, peanuts and blueberries. ${ }^{33}$ Its association with the benefits of CRis correlated with a subsequent increase in activity of PGC- $1 \alpha$, which can increase mitochondrial mass and biogenesis in liver, muscle and brain. ${ }^{34,35}$ This increase in PGC- $1 \alpha$ is through administration of SRT1720, an analog of resveratrol 1000times stronger than the polyphenol itself. An analysis comparing gene expression from $\mathrm{CR}$ versus induction via resveratrol analog SRT1720 was comparable, significantly shifting in the same direction. Furthermore, analysis indicated attenuation of PPAR as well as NRIP1 (an inhibitor of PGC-1 $\alpha$ ) both from the same mechanism of action. ${ }^{36}$ On the other hand, there is a lack of studies that suggest any further significant benefit of resveratrol similar to that of CR including but not limited to: weight loss, glucose/insulin homeostasis and reduction of chronic disease risk.

Metformin is an oral hypoglycemic agent used in the treatment of diabetes to decrease hepatic glucose production and potentiate the effect of insulin. ${ }^{37}$ In this, the risk of glucotoxic effects such as glycosylation and oxidative damage are reduced. The correlations between metformin and $\mathrm{CR}$ include expression of glycolytic genes, reduction of ROS generation and oxidative accumulation and activation of AMPK (involved in PGC- $1 \alpha$ regulation). ${ }^{38,39}$ Interestingly, metformin compromises production of ATP, which results in inhibition of a mitochondrial complex; this inhibition is a known cause of oxidative damage. ${ }^{40,41}$ Furthermore, AMPK is part of a larger regulatory pathway and but one of the external signals involved in PGC- $1 \alpha$ regulation. It alone cannot be determined to have all of the benefits associated with CR; additionally, AMPK is found to increase PGC- $1 \alpha$ and PPAR under starvation conditions. ${ }^{36}$

\section{Discussion}

The research behind calorie restriction and its impact on longevity and health span are numerous and controversial. The majority of current research indicates a positive correlation with CR resulting in alterations in epigenetic markers, decrease in oxidative stress through ROS and reduction in chronic disease risk and metabolic syndrome. Animal and human studies have suggested significant changes in all of these areas; however, additional human research needs to take place in order to truly examine the efficacy of CR on reduction of DNA damage and its correlation with longevity related biomarkers. Despite the evident promise of beneficial clinical outcomes, human studies are limited and of short duration; thus, it is impossible to conclude such benefits in a human population. Furthermore, options being explored to mimic the effects of $\mathrm{CR}$ are numerous and are far more enticing to the general public than CR. As further human studies with longer durations arise, it will be interesting to see the conclusions that are formed and what clinical recommendations are formulated.

\section{Conclusion/Clinical implications}

At this time, there are limited to no clinical implications that can be drawn from the current body of research. Modest calorie intake and weight loss are already well-established clinical recommendations used to promote health and reduce chronic disease risk; however, the intentional reduction of caloric intake is not adequately well founded to provide the basis for public health or personal recommendations. Investigating the efficacy of $\mathrm{CR}$ on non-overweight individuals or those who are morbidly obese has not yet been seen. In addition, research of CR in populations where longevity and health span are of utmost concern, namely the elderly, is non-existent. Weight loss in elderly individuals of healthy weight may be indicated as harmful, especially in those who are frail or suffer from sarcopenia. In this case, the risks may outweigh the potential benefits. Thus, further human studies need to be conducted before any clinical or public health recommendations can be determined.

\section{Acknowledgements}

None.

\section{Conflict of interest}

The author declares no conflict of interest.

\section{References}

1. Frye RA. Characterization of five human cDNAs with homology to the yeast SIR2 gene: Sir2-like proteins (sirtuins) metabolize NAD and may have protein ADP-ribosyltransferase activity. Biochem Biophys Res Commun. 2003;260(1):273-279.

2. Sinclair DA, Guarente L. Unlocking the Secrets of Longevity Genes. Sci Am. 2006;294(3):48-51.

3. Bordone L, Guarente L. Calorie Restriction, Sirt1 and Metabolism: understanding longevity. Nat Rev Mol Cell Biol. 2005;6(4):298-305.

4. Cacciapuoti F. Opposite effects of metabolic syndrome and calorie restriction on thrombotic disease: heads and tails of the same coinresveratrol's role. Metab Syndr Relat Disord. 2009;7(5):397-400.

5. Lin SJ, Kaeberlein M, Andalis AA, et al. Calorie restriction extends saccharomyces cerevisiae lifespan by increasing respiration. Nature. 2002;418(6895):344-348.

6. Nemoto S, Fergusson MM, Finkel T. Nutrient availability regulates SIRT1 through a forkhead-dependent pathway. Science. 2004;306(5704):21052108.

7. Cohen HY, Miller C, Bitterman KJ, et al. Calorie restriction promotes mammalian cell survival by inducing the SIRT1 deacetylase. Science. 2004;305:390-392.

8. Mattson MP, Duan W, Guo Z. Meal size and frequency affect neuronal plasticity and vulnerability to disease: cellular and molecular mechanisms. J Neurochem. 2003;84(3):417-431.

9. Canty, David. Advanced glycation end products, Protein, Fats and Carbohydrates. USA; 2013.

10. Lal Mark A, Brismar H, Elkof Ac, et al. Role of oxidative stress in advanced glycation end product-induced mesangial cell activation. Kidney Int. 2002;61(6):2006-2014.

11. Davies KJ. Oxidative stress: the paradox of aerobic life. Biochem Soc Symp. 1995;61:1-31.

12. Raha S, Robinson BH. Mitochondria, oxygen free radicals, disease and aging. Trends Biochem Sci. 2000;25(10):502-508.

13. Kim HJ, Jung KJ, Yu BP, et al. Modulation of redox-sensitive transcription factors by calorie restriction during aging. Mech Ageing Dev. 2002;123(12):1589-1595.

14. Speakman JR, Talbot DA, Selman C, et al. Uncoupled and surviving: individual mice with high metabolism have greater mitochondrial uncoupling and live longer. Aging Cell. 2004;3:87-95. 
15. Rooyackers OE, Adey DB, Ades PA, et al. Effect of age on in vivo rates of mitochondrial protein synthesis in human skeletal muscle. Proc Natl Acad Sci. 1996;93(26):15364-15369.

16. Wanagat J, Cao Z, Pathare $\mathrm{P}$, et al. Mitochondria DNA deletion mutations colocalize with segmental electron transport system abnormalities, muscle fiber atrophy, fiber splitting and oxidative damage in sarcopenia FASEB J. 2001;15(2):322-332.

17. Bevilacqua L, Ramsey JJ, Hagopian K, et al. Effects of short- and medium-term calorie restriction on muscle mitochondrial proton leak and reactive oxygen species production. Am J Physiol Endocrinol Metab. 2004;286(5):E852-E861.

18. Faulks SC, Turner N, Else PL, et al. Calorie restriction in mice: effects on body composition, daily activity, metabolic rate, mitochondrial reactive oxygen species production and membrane fatty acid composition. $J$ Gerontol A Biol Sci Med Sci. 2006;61(8):781-794.

19. Baker DJ, Betik AC, Krause DJ, et al. No decline in skeletal muscle oxidative capacity with aging in long-term calorically restricted rats:effects are independent of mitochondrial DNA integrity. J Gerontol A Biol Sci Med Sci. 2006;61(7):675-684.

20. Heilbronn LK, de Jonge L, Frisard ML, et al. Effect of 6-month calorie restriction on biomarkers of longevity, metabolic adaptation and oxidative stress in overweight individuals: a randomized controlled trial. JAMA. 2006;295(13):1539-1548.

21. Chiribau $\mathrm{CB}$, Cheng L, Cucoranu IC, et al. FOXO3A regulates peroxiredoxin III expression in human cardiac fibroblasts. $\mathrm{J}$ Biol Chem. 2008;283(13):8211-8217.

22. Marinkovic D, Zhang X, Yalcin S, et al. FOXO3 is required for the regulation of oxidative stress in erythropoiesis. J Clin Invest. 2007;117(8):2133-2144.

23. Kenyon C, Chang J, Gensch E, et al. Elegans mutant that lives twice as long as wild type. Nature. 1993;366(6454):461-464.

24. Woods SC. Signals that influence food intake and body weight. Physiol Behav. 2005;86(5):709-716.

25. Colman RJ, Anderson RM, Johnson SC, et al. Calorie Restriction Delays Disease Onset and Mortality in Rhesus Monkeys. Science. 2009;325(5937):201-204.

26. Mattison JA, Roth GS, Beasley TM, et al. Impact of caloric restriction on health and survival in rhesus monkeys from the NIA study. Nature. 2012;489(7415):318-322.

27. Ingram DK, Zhu M, Mamczarz, et al. Calorie restriction mimetics: an emerging research field. Aging Cell. 2006;5(2):97-108.
28. Weindruch R, Walford RL, Fligiel S, et al. The Retardation of Aging in Miceby Dietary Restriction: Longevity, Cancer, Immunity and Lifetime Energy Intake. J Nutr. 1986;116(4):641-654.

29. Speakman JR, Hambly C. Starving for Life: what animal studies can and cannot tell us about the use of calorie restriction to prolong human lifespan. J Nutr. 2007;137(4):1078-1086.

30. Masoro EJ. Hormesis and the antiaging action of dietary restriction. Exp Gerontol. 1998;33(1-2):61-66.

31. Klein S, Fontana L, Young VL, et al. Absense of an effect of liposuction on insulin action and risk factors for coronary artery disease. $N$ Engl J Med. 2004;350(25):2549-2557.

32. Muzumdar R, Allison DB, Huffman DM, et al. Visceral adipose tissue modulates mammalian longevity. Aging Cell. 2008;7(3):438-440.

33. The Mayo Clinic. Red Wine and Resveratrol. 2011.

34. Baur JA, Pearson JK, Price NL, et al. Resveratrol improves health and survival of mice on a high-calorie diet. Nature. 2006;444(7117):337342

35. Lagouge M, Argmann C, Gerhart-Hines Z, et al. Resveratrol improves mitochondrial function and protects against metabolic disease by activating SIRT1 and PGC-1alpha. Cell. 2006;127:1109-1122.

36. Martin-Montalvo A, de Cabo R. Mitochondrial Metabolic Reprogramming induced by Calorie Restriction. Antioxid Redox Signal. 2013;19(3):310-320.

37. Pronsky Z. Food-Medication Interactions. Birchrunville, PA, USA 2011.

38. Algire C, Moiseeva O, Deschênes-Simard X, et al. Metformin reduces endogenous reactive oxygen species and associated DNA damage. Cancer Prev Res. 2012;5(4):536-543.

39. Canto C, Auwerx J. Calorie restriction: is AMPK a key sensor and effector? Physiology. 2011;26(4):214-224.

40. Hariharakrishnan J, Satpute RM, Prasad GB, et al. Oxidative stress mediated cytotoxicity of cyanide in LLC-MK2 cells and its attenuation by alpha-ketoglutarate and $\mathrm{N}$-acetyl cysteine. Toxicol Lett. 2009;185(2):132-141.

41. Testa CM, Sherer TB, Greenamyre JT. Rotenone induces oxidative stress and dopaminergic neuron damage in organotypic substantia nigra cultures. Brain Res Mol Brain Res. 2005;134(1):109-118. 